\title{
A Result on Line Graphs and Hamiltonian Graphs
}

\author{
S.Venu Madhava Sarma \\ Assistant Professor in \\ Mathematics, K.L.UNIVERSITY \\ Vaddeswaram Post,Guntur
}

\author{
T.Ravi Kumar \\ Associate Professor in Computer \\ Science, K.L.UNIVERSITY \\ Vaddeswaram Post, Guntur
}

\author{
T.V.Pradeep Kumar \\ Assistant Professor in \\ Mathematics, Acharya Nagarjuna \\ University, Guntur
}

\begin{abstract}
In 1856, Hamiltonian introduced the Hamiltonian Graph where a Graph which is covered all the vertices without repetition and end with starting vertex. In this Paper I would like to prove that

If ' $\mathrm{G}$ ' is a Complete and locally Complete graph, on $\mathrm{n} \geq 3$ vertices, which does not contain an induced $K_{1,3}$, then $G$ is Hamiltonian.
\end{abstract}

Keywords: Graph, Hamiltonian Graph, Complete Graph, Neighborhood, Locally Complete Graph.

\section{INTRODUCTION}

Graphs, considered here, are finite, undirected and simple and complete Graphs being followed for terminology and notation. let $G=(V, E)$ be a graph, with $V$ the set of vertices and $E$ the set of edges. Suppose that $W$ is a nonempty subset of $V$. The sub graph of $G$, whose vertex set is $W$ and whose edge set is the set of those edges of $G$ that have both ends in $W$, is called the sub graph of $G$ induced by $W$ and is denoted by $G[W]$. For any vertex $v$ in $V$, the neighbour set of $v$ is the set of all vertices adjacent to $v$. This set is denoted by $N(v)$. For a graph $G=(V, E)$, we shall denote

$$
\begin{aligned}
& \delta(G)=\min |\mathrm{N}(\mathrm{v})| \\
& v \in V \quad \Delta(\mathrm{G})=\underset{v \in V}{\max }|\mathrm{N}(\mathrm{v})|
\end{aligned}
$$

a graph $\mathrm{G}=(\mathrm{V}, \mathrm{E})$ is locally complete, if for each vertex $v$ the graph $\mathrm{G}[\mathrm{N}(v)]$ is complete. With every graph $\mathrm{G}$, having at least one edge, there exists associated a graph $\mathrm{L}(\mathrm{G})$, called the line graph of $\mathrm{G}$, whose vertices, can be put in a one-to-one correspondence with the edges of $G$, in such a way that two vertices of $L(G)$ are adjacent if and only if the corresponding edges of $G$ are adjacent.

The neighborhood is often denoted $N_{G}(v)$ or (when the graph is unambiguous) $N(v)$. The same neighborhood notation may also be used to refer to sets of adjacent vertices rather than the corresponding induced sub graphs. The neighborhood described above does not include $v$ itself, and is more specifically the open neighborhood of $v$; it is also possible to define a neighborhood in which $v$ itself is included, called the closed neighborhood and denoted by $N_{G}[v]$. When stated without any qualification, a neighborhood is assumed to be open.

1.1 Definition: A graph - usually denoted $G(V, E)$ or $G=$ $(\mathrm{V}, \mathrm{E})$ - consists of set of vertices $\mathrm{V}$ together with a set of edges $E$. The number of vertices in a graph is usually denoted $n$ while the number of edges is usually denoted $m$.
1.2 Definition: Vertices are also known as nodes, points and (in social networks) as actors, agents or players.

1.3 Definition: Edges are also known as lines and (in social networks) as ties or links. An edge

$\mathrm{e}=(\mathrm{u}, \mathrm{v})$ is defined by the unordered pair of vertices that serve as its end points.

1.4 Example: The graph depicted in Figure 1 has vertex set $\mathrm{V}=\{\mathrm{a}, \mathrm{b}, \mathrm{c}, \mathrm{d}, \mathrm{e} . \mathrm{f}\}$ and edge set

$E=\{(a, b),(b, c),(c, d),(c, e),(d, e),(e, f)\}$.

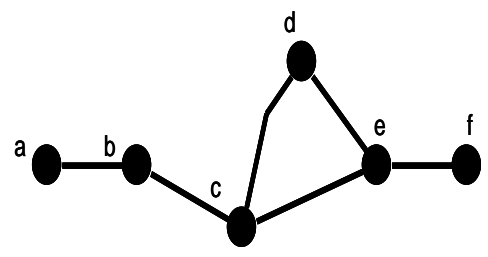

Figure 1.

1. 5 Definition: Two vertices $u$ and $v$ are adjacent if there exists an edge $(u, v)$ that connects them.

1.6 Definition: An edge (u,v) is said to be incident upon nodes $\mathrm{u}$ and $\mathrm{v}$.

1.7 Definition: An edge $\mathrm{e}=(\mathrm{u}, \mathrm{u})$ that links a vertex to itself is known as a self-loop or reflexive tie.

1.8 Definition: Every graph has associated with it an adjacency matrix, which is a binary $n \times n$ matrix $\mathrm{A}$ in which $\mathrm{a}_{\mathrm{ij}}=1$ and $\mathrm{a}_{\mathrm{ji}}=$ 1 if vertex vi is adjacent to vertex vj, and aij $=0$ and aji $=0$ otherwise. The natural graphical representation of an adjacency matrix is a table, such as shown below.

\begin{tabular}{lllllll}
\multicolumn{1}{c}{} & $\mathrm{a}$ & $\mathrm{b}$ & $\mathrm{c}$ & $\mathrm{d}$ & $\mathrm{e}$ & $\mathrm{f}$ \\
$\mathrm{a}$ & 0 & 1 & 0 & 0 & 0 & 0 \\
$\mathrm{~b}$ & $\mathrm{l}$ & 0 & 1 & 0 & 0 & 0 \\
$\mathrm{c}$ & 0 & 1 & 0 & 1 & 1 & 0 \\
$\mathrm{~d}$ & 0 & 0 & 1 & 0 & 1 & 0 \\
$\mathrm{e}$ & 0 & 0 & 1 & 1 & 0 & 1 \\
$\mathrm{f}$ & 0 & 0 & 0 & 0 & 1 & 0 \\
\hline
\end{tabular}

Adjacency matrix for graph in Figure 1. 
1.9 Definition: Examining either Figure 1 or given adjacency Matrix, we can see that not every vertex is adjacent to every other. A graph in which all vertices are adjacent to all others is said to be complete.

1.10 Definition: While not every vertex in the graph in Figure 1 is adjacent, one can construct a sequence of adjacent vertices from any vertex to any other. Graphs with this property are called connected.

1.11 Note: Reachability. Similarly, any pair of vertices in which one vertex can reach the other via a sequence of adjacent vertices is called reachable. If we determine reachability for every pair of vertices, we can construct a reachability matrix $\mathrm{R}$ such as depicted in Figure 2. The matrix $\mathrm{R}$ can be thought of as the result of applying transitive closure to the adjacency matrix A.

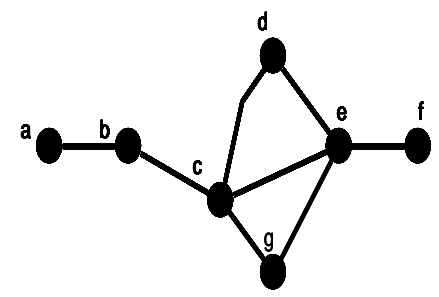

Figure: 2

1.12 Definition : A walk is closed if $\mathrm{v}_{\mathrm{o}}=\mathrm{v}_{\mathrm{n}}$.degree of the vertex and is denoted $\mathrm{d}(\mathrm{v})$.

1.13 Definition : A tree is a connected graph that contains no cycles. In a tree, every pair of points is connected by a unique path. That is, there is only one way to get from A to B.

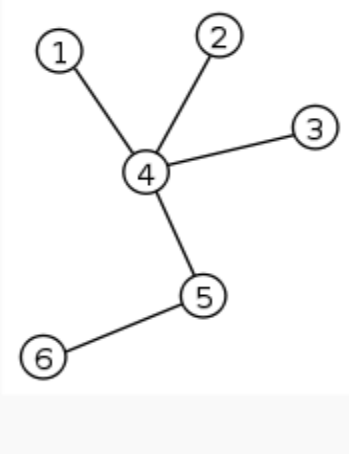

Figure 3: A labeled tree with 6 vertices and 5 edges

1.14 Definition: A spanning tree for a graph $G$ is a sub-graph of $G$ which is a tree that includes every vertex of $G$.

1.15 Definition: The length of a walk (and therefore a path or trail) is defined as the number of edges it contains. For example, in Figure 3, the path $a, b, c, d, e$ has length 4.

1.16 Definition: The number of vertices adjacent to a given vertex is called the degree of the vertex and is denoted $\mathrm{d}(\mathrm{v})$.
1.17 Definition : In the mathematical field of graph theory, a bipartite graph (or bigraph) is a graph whose vertices can be divided into two disjoint sets $U$ and $V$ such that every edge connects a vertex in $U$ to one in $V$; that is, $U$ and $V$ are independent sets. Equivalently, a bipartite graph is a graph that does not contain any odd-length cycles.

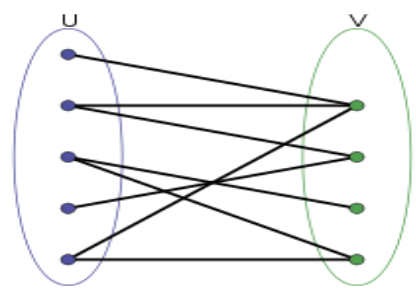

Figure 4: Example of a bipartite graph.

1.18 Definition : An Eulerian circuit in a graph $G$ is circuit which includes every vertex and every edge of G. It may pass through a vertex more than once, but because it is a circuit it traverse each edge exactly once. A graph which has an Eulerian circuit is called an Eulerian graph. An Eulerian path in a graph $\mathrm{G}$ is a walk which passes through every vertex of $\mathrm{G}$ and which traverses each edge of $\mathrm{G}$ exactly once

1.19 Example : Königsberg bridge problem: The city of Königsberg (now Kaliningrad) had seven bridges on the Pregel River. People were wondering whether it would be possible to take a walk through the city passing exactly once on each bridge. Euler built the representative graph, observed that it had vertices of odd degree, and proved that this made such a walk impossible. Does there exist a walk crossing each of the seven bridges of Königsberg exactly once
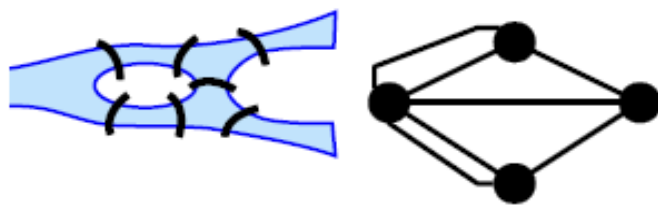

Figure 5: Konigsberg problem

\section{COMPLETE GRAPHS, LOCALLY COMPLETE GRAPHS, HAMILTONIAN GRAPHS, LINE GRAPHS}

In this section we have to prove that main theorem using definitions.

2.1 Definition: A Hamilton circuit is a path that visits every vertex in the graph exactly once and return to the starting vertex. Determining whether such paths or circuits exist is an NP-complete problem. In the diagram below, an example Hamilton Circuit would be 


\subsection{Example :}

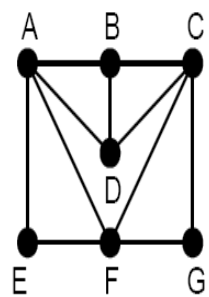

Figure 6: Hamilton Circuit would be AEFGCDBA.

2.3 Definition : Compete Graph: A simple graph in which there exists an edge between every pair of vertices is called a complete graph.

2.4 Definition : Let $\{v 1, v 2 \ldots \ldots v n\}$ be the vertex set of a graph $\mathrm{G}$, and for each ' $\alpha$ '.

let $N i$ "denote the closed neighborhood of $v_{\mathrm{a}}$. Let $N_{\mathrm{a}}$ be any subset of $\mathrm{N}_{a}{ }^{*}$ containing $v_{a}$ which generates a complete subgraph $C_{a}$ of $\mathrm{G}$. Then $C_{a}$ is called a complete sub neighborhood of $v_{a}$, and the indexed family $C^{*}=\left\{C_{1}, C_{2}, \ldots\right.$, $\left.C_{\text {n }}\right\}$ is called a complete family for $\mathrm{G}$ if $\mathrm{G}=\bigcup \mathrm{C}^{*}$. A graph $\mathrm{G}$ is called locally complete iff $\mathrm{G}$ has at least one complete family.

2.5 Examples : It is easily seen that complete graphs, trees, and unicyclic graphs are also locally complete.

The complete bigraph $K_{3,2}$ is the smallest (nontrivial, connected) graph which fails to be locally complete.

2.6 Theorem : If ' $G$ ' is a Complete and locally Complete graph, on $\mathrm{n} \geq 3$ vertices, which does not contain an induced $\mathrm{K}_{1,3}$, then $\mathrm{G}$ is Hamiltonian.

Proof : Suppose that the Theorem is not true

let 'G' be a complete and locally Complete graph on at least three vertices,

which does not contain an induced $\mathrm{K}_{1,3}$, but which is not Hamiltonian.

Clearly, 'G' contains a cycle.

Let ' $\mathrm{C}$ ' be a largest cycle in ' $\mathrm{G}$ '.

Then, ' $C$ ' does not span ' $G$ ' and, since ' $G$ ' is complete, there exists a vertex $v$, not on ' $C$ ', which is adjacent to a vertex $u$, lying on ' $\mathrm{C}$ '.

Let $\mathrm{u}_{1}$ and $\mathrm{u}_{2}$ be the vertices neighbouring ' $\mathrm{u}$ ', on the cycle 'C'.

Since ' $G$ ' is locally complete, there exists a path ' $P$ ', in $\mathrm{G}[\mathrm{N}(\mathrm{u})]$,

From ' $v$ ' to the one of $u_{1}$ or $u_{2}$,

which does not include the other.

Without loss of generality,

we shall suppose that ' $\mathrm{P}$ ' is a path from ' $v$ ' to $\mathrm{u}_{1}$ and that $\mathrm{u}_{2}$ $\notin \mathrm{P}$.

Now,

if $P \cap C=\left\{\mathrm{u}_{1}\right\}$, then, by attaching ' $\mathrm{P}$ ' to ' $\mathrm{C}$ ' at $\mathrm{u}_{1}$ and $v$,

we could obtain a cycle larger than $\mathrm{C}$.

Hence, we may assume that $\mathrm{P} \cap \mathrm{C}$ contains vertices other than $\mathrm{u}_{1}$.

Also, we cannot have ' $v$ ' adjacent to either $\mathrm{u}_{1}$ or $\mathrm{u}_{2}$, without producing

a cycle larger than ' $\mathrm{C}$ '.

Thus, since $\left\{u, u_{1}, u_{2}, v\right\}$ cannot induce a $K_{1,3}$ in ' $G$ ', then it must be that $u_{1} u_{2}$ is an edge of ' $G$ '.

For the purpose of this proof,

we shall define a singular vertex to be a vertex

$\mathrm{w} \in \mathrm{P} \cap \mathrm{C}-\{\mathrm{u} 1\}$, such that neither of the vertices, neighboring ' $\mathrm{w}$ ' in $\mathrm{C}$, belongsto $\mathrm{N}(\mathrm{u})$.

\section{We shall consider two cases:}

Case 1. Every vertex in $P \cap C-\left\{u_{1}\right\}$ is singular.

Then, for any vertex

$\mathrm{w} \in \mathrm{P} \cap \mathrm{C}-\left\{\mathrm{u}_{1}\right\}, \mathrm{w}$ is adjacent to $\mathrm{u}$, but neither of the vertices $\mathrm{w}_{1}$ and $\mathrm{w}_{2}$,

neighboring ' $\mathrm{w}$ ' on ' $\mathrm{C}$ ', belongs to $\mathrm{N}(\mathrm{u})$.

Thus, since $\left\{\mathrm{w}, \mathrm{w}_{1}, \mathrm{w}_{2}, \mathrm{u}\right\}$ cannot induce a $\mathrm{K}_{1,3}$ in ' $\mathrm{G}$ ', then it must be that $\mathrm{w}_{1} \mathrm{w}_{2}$ is an edge of ' $\mathrm{G}$ '.

Now, traverse $\mathrm{C}$, starting at $\mathrm{u}_{2}$ and moving away from $\mathrm{u}$ and for each vertex

$\mathrm{w} \in \mathrm{P} \cap \mathrm{C}-\left\{\mathrm{u}_{1}\right\}$, by-pass $\mathrm{w}$, by taking the edge $\mathrm{w}_{1} \mathrm{w}_{2}$.

Continue, until the vertex $\mathrm{u}_{1}$ is reached. Then, follow $\mathrm{P}$ from $\mathrm{u}_{1}$ to $v$ then to $\mathrm{u}$ and finish at $\mathrm{u}_{2}$.

Then, we have passed through each vertex of $\mathrm{C} \cup \mathrm{P}$, exactly once, and have

thus constructed a cycle larger than $\mathrm{C}$.

Case 2: $\mathrm{P} \cap \mathrm{C}-\{\mathrm{u} 1\}$ contains non singular vertices.

Then, follow $\mathrm{P}$ from $v$ toward $\mathrm{u}_{1}$, until the first nonsingular vertex $\mathrm{w}$ is reached. Let $\mathrm{w}_{1}$ and $\mathrm{w}_{2}$ be the vertices neighbouring $\mathrm{w}$ along $\mathrm{C}$.

Then, at least one of $\mathrm{w}_{1}$ and $\mathrm{w}_{2}$ is adjacent to $\mathrm{u}$.

Without loss of generality, suppose that $\mathrm{w}_{1}$ is adjacent to $\mathrm{u}$.

Now,

form a new cycle $\mathrm{C}^{1}$, containing exactly the same vertices as $\mathrm{C}$, as follows.

Delete the edges ww1, uu1 and uu2 and add the edges $w u, w_{1} u$ and $\mathrm{u}_{1} \mathrm{u}_{2}$.

Note that if $\mathrm{w}$ is a neighbour of $\mathrm{u}_{1}$ or $\mathrm{u}_{2}$, then not all of these edges may be distinct

(e.g., if $w 1=u 1$, then $u u 1=u w 1$ ).

But now, the vertices neighbouring $\mathrm{u}$ in $\mathrm{C}^{1}$ are $\mathrm{w}$ and $\mathrm{w} 1$, and the subpath $\mathrm{P}^{1}$ of $\mathrm{P}$, from $\mathrm{w}$ to $v$, does not include $\mathrm{w}_{1}$

(as else w1, being a nonsingular vertex, would have been chosen earlier, instead of w).

Moreover, from the choice of $\mathrm{w}$, it follows that $\mathrm{P}^{1}$ cannot contain any 
Non singular vertex with respect to $\mathrm{C}^{1}$ and $\mathrm{w}$ (in the place of u1). Thus, relative

to $\mathrm{P}^{1}$ and $\mathrm{C}^{1}$, we are back to the Case 1 . Hence, in any case, $\mathrm{C}$ cannot have

been a largest cycle and, with this contradiction, Hence the Theorem is proved.

Remark. The above Theorem does not provide a necessary condition. For example,

let us consider the graph $\mathrm{G}=(\mathrm{V}, \mathrm{E})$, where

$\mathrm{V}=\left\{v_{1}, v_{2}, \ldots, v_{6}\right\}$

and $E=\left\{v_{1} v_{2}, v_{1} v_{6}, v_{2} v_{3}, v_{2} v_{4}, v_{2} v_{5}, v_{2} v_{6}, v_{3} v_{4}, v_{3} v_{6}, v_{4} v_{5}, v_{4} v_{6}\right.$, $\left.v_{5} v_{6}\right\}$.

Obviously, this graph is Complete, locally Complete, Hamiltonian, but

$\mathrm{G}\left[\left\{v_{1}, v_{2}, v_{3}, v_{5}\right\}\right]$ is isomorphic to $\mathrm{K}_{1,3}$.

If $L(G)$ is the line graph of a graph ' $G$ ', then it is well known that $\mathrm{L}(\mathrm{G})$

cannot contain $\mathrm{K} 1,3$ as an induced sub graph. Thus, we have the following

Corollary 1. Every complete and locally complete line graph, on $\mathrm{n} \geq 3$

vertices, is Hamiltonian.

Corollary 2. If every edge of a complete graph ' $\mathrm{G}$ ' lies in a triangle, then $\mathrm{L}(\mathrm{G})$

is Hamiltonian.

Proof. If every edge of $\mathrm{G}$ lies in a triangle, then $\mathrm{L}(\mathrm{G})$ is locally complete and

by Corollary $1, \mathrm{~L}(\mathrm{G})$ is Hamiltonian.

Corollary 3. If $\mathrm{G}$ is a complete and locally complete graph, on $n \geq 3$ vertices, then $L(G)$ is Hamiltonian.

Proof. If $\mathrm{G}$ is complete and locally complete, on at least three vertices,

then every edge of $\mathrm{G}$ must lie in a triangle and, hence, $\mathrm{L}(\mathrm{G})$ is Hamiltonian

Corollary 4. If $\mathrm{G}$ is a complete graph with $\delta(\mathrm{G}) \geq 3$, then $\mathrm{L}(\mathrm{L}(\mathrm{G}))$ is Hamiltonian.

Corollary 5.If $\mathrm{G}$ is Hamiltonian, then $\mathrm{L}(\mathrm{G})$ is Hamiltonian.

Proof : This is a nice, basic result to see if a line graph is Hamiltonian. A graph is
Hamiltonian if there exists a Hamiltonian cycle in the graph It may be easier to find a Hamiltonian cycle in $G$ than $L(G)$, but from this proposition, we would get that $\mathrm{L}(\mathrm{G})$ is Hamiltonian.

\section{REFERENCES}

[1] Bondy J.A. and V. Chvátal, A Method in Graph Theory, Discr. Math. 15 1976), pp 111-136.

[2] Dirac G.A., Some Theorems on Abstract Graphs, Proc. Lond. Math. Soc. 2 (1952), pp 69-81.

[3] Garey M.R and D.S. Jhonson, Computers and Intractability: A Guide to the Theory of NPCompleteness, W.H. Freeman and Company, New York.

[4] O. Ore, Note on Hamiltonian Circuits, Am. Mat. Monthly 67 (1960), pp 55.

[5] West D.B, Introduction to Graph Theory, Prentice-Hall, Inc., New Jersey.

[6] Williams - Nash, C.St.J.A. "Hamiltonian Arcs and Circuits" in recent trends in graph theory ed. By M. Capobianco et al., Springer - verlag, Berlin, 1971

[7] V.Chvatal "New directions in Hamiltonian Graph theory" In New directions in graph theory ed. By F.Harary, Academic press, N.Y.London, 1973.

[8] Venu Madhava Sarma.S and T.V. Pradeep Kumar International Journal of Mathematical Archive-2(12), 2011,Page 2538-2542.

[9] Venu Madhava Sarma.S International Journal of Computer Application, Issue 2, Volume 1(February 2012), Page 21-31.

[10] Venu Madhava Sarma.S and T.V.Pradeep Kumar International J. of Math. Sci. \& Engg. Appls. (IJMSEA) ISSN 0973-9424, Vol. 6 No. III (May, 2012), pp. 47-54.

[11] Venu Madhava Sarma.S and T.V.Pradeep Kumar proceedings of Two Day UGC National seminar on "Modern Trends in Mathematics and Physical Sciences ( NSMTMPS - 2012) dated 20 ${ }^{\text {th }}, 21$ st Jan, 2012.

[12] D. P. Geller, The square root of a digraph, J. Combinatorial Theory, 5 (1968), 320-321.

[13] F. Harary, Graph Theory , Addison-Wesley, Reading, Mass., 1969.

[14] A. Mukhopadhyay, The square root of a graph, J. Combinatorial Theory, 2 (1967), 290-295. 